\title{
BREAKDOWN OF TIME-REVERSAL SYMMETRY: INTRINSIC RANDOMNESS, TIME OPERATOR, SCATTERING
}

\author{
FERNANDO GÓMEZ-CUBILLO AND ZDZISLAW SUCHANECKI
}

Communicated by Arno Bohm

\begin{abstract}
Misra-Prigogine-Courbage theory of irreversibility is revisited on the basis of Nagy-Foiaş dilation theory and Halmos-Helson theory of invariant subspaces. Universal models for intrinsically random dynamics are given as well as equivalent conditions to the existence of internal time operators, where innovation processes and Lax-Phillips scattering appear in a natural way.
\end{abstract}

\section{Introduction}

The problem of reconciling the apparent irreversible behavior of (macroscopic) systems with the reversible nature of fundamental microscopic laws of physics, including both classical and quantum mechanics, is far from being completely solved. Even experimental evidences of the irreversible behavior at microscopic level associated to chaotic behavior and with no requirement that the dynamical equations violate time-reversal symmetry or the system be coupled to a source of external noise have been recently found [9]. In the late 1970's Misra, Prigogine and Courbage (MPC) [7,8] already discussed the question of the dynamical meaning of the second law of thermodynamics at microscopic level expressing intrinsic irreversibility in terms of the existence of Lyapounov operators - i.e., observables varying monotonically in time - and their close links with the inherent randomness of the system, its dynamical instability - for instance, mixing property is necessary - and the existence of internal time operators.

This work is a mathematical approach to MPC theory of irreversibility in the context of statistical mechanics. Universal models for intrinsically random dynamics are given in Section 2 on the basis of Nagy-Foiaş dilation theory [10]. Equivalent conditions to the existence of internal time operators are derived in Section 3 in terms of Halmos-Helson theory of invariant subspaces [4,5], where innovation processes [11] and Lax-Phillips scattering [6] appear as alternative descriptions. 


\section{Intrinsic Randomness}

Let us consider abstract dynamical systems $\left(\Omega, \mathcal{A}, \mu,\left\{S_{t}\right\}\right)$, where $\Omega$ is the phase space of the system equipped with the $\sigma$-algebra $\mathcal{A}$ and $\left\{S_{t}\right\}$ is a group of measurable point transformations on $\Omega$ preserving the probability measure $\mu$ (automorphic case). As usual in statistical mechanics and ergodic theory, trajectories are replaced by density functions (states $\rho$ ) and $\left\{S_{t}\right\}$ by the induced group $\left\{U_{t}\right\}$ of unitary operators in $L^{2}=L^{2}(\Omega, \mathcal{A}, \mu)$ given by

$$
U_{t} \rho(\omega):=\rho\left(S_{-t} \omega\right), \quad \rho \in L^{2} .
$$

Each $U_{t}$ preserves positivity ( $\rho \geq 0$ implies $\left.U_{t} \rho \geq 0\right)$ and normalization $\left(\int \rho d \mu=\right.$ $\int U_{t} \rho d \mu=1$, for states $\rho$ ), and $U_{t} 1=1$. On the other hand, every Markov process on $\Omega$ with stationary distribution $\mu$ is associated with a semigroup of contractions $\left\{W_{t}\right\}$ on $L^{2}$ which also preserves positivity and satisfies $W_{t} 1=1$, where now the constant density 1 is the equilibrium state. From the point of view of the second law of thermodynamics, we are interested only in irreversible Markov processes which correspond to monotonic Markov semigroups, i.e.,

$$
\left\|W_{t} \rho-1\right\| \text { decreases monotonically to } 0 \text { as } t \rightarrow \infty
$$

for all states $\rho \neq 1$.

MPC demonstrated the possibility of obtaining stochastic Markov processes from deterministic dynamics simply through a change of representation which involves no loss of information provided the dynamical system under consideration has a suitably high degree of instability of motion. MPC intrinsic randomness is based on the existence of positivity preserving quasi-affinities $\Lambda$ relating unitary groups $\left\{U_{t}\right\}$ to monotonic Markovian semigroups $\left\{W_{t}\right\}$ on $L^{2}$ by means of an intertwining relation of the form

$$
W_{t} \Lambda=\Lambda U_{t}, \quad t \geq 0 .
$$

In such case, the deterministic dynamics $\left\{U_{t}\right\}$ is said to be intrinsically random and $\left\{W_{t}\right\}$ is called a random image of $\left\{U_{t}\right\}$. For intrinsically random dynamics $M=\Lambda^{*} \Lambda$ is a Lyapounov operator. Here by a quasi-affinity we mean a linear, one-to-one and continuous transformation $\Lambda$ from $L^{2}$ onto a dense subspace of $L^{2}$, so that $\Lambda^{-1}$ exists on this dense domain, but is not necessarily continuous. Relation (2) implies that $\Lambda$ preserves positivity and normalization, and $\Lambda 1=1$. In what follows the superfluous one-dimensional subspace of $L^{2}$ spanned by the constant functions shall be denoted by $\mathbb{C}$. 
Universal models for intrinsically random unitary dynamics can be given in terms of Nagy-Foiaş dilation theory and the corresponding functional calculus [10]. For it, recall that every contraction $W$ on $L^{2}$ has a minimal isometric dilation $U_{+}$on some Hilbert space $\mathcal{K}_{+} \supset L^{2}$, determined up to isomorphism. Being an isometry, $U_{+}, \mathcal{K}_{+}$admits a unique Wold decomposition [10, Theorem I.1.1] into a unitary part and a unilateral shift, where the unitary part $R, \mathcal{R}$ is given by

$$
R:=U_{+} \mid \mathcal{R}, \quad \mathcal{R}:=\bigcap_{n=0}^{\infty} U_{+}^{n} L^{2}
$$

and is called the residual part of $U_{+}, \mathcal{K}_{+}$. In particular, if the cogenerator of a random image $\left\{W_{t}\right\}$ on $L^{2}$, i.e., is the Cayley transform, i.e., $W=(A+I)(A-$ $I)^{-1}$ of the infinitesimal generator $A$ of $\left\{W_{t}\right\}$, then $R$ is the cogenerator of the unitary group $\left\{R_{t}\right\}$ on $\mathcal{R}$, where $R_{t}$ is the residual part corresponding to $W_{t}$ for each $t \geq 0$. See [10, Section III.8-9] for details. In what follows we assume that ker $W=\{0\}$. Next theorem states that residual groups are universal models for intrinsically random unitary dynamics, whereas random images must belong to a concrete class of contraction semigroups [2]

Theorem 1. Let $\left\{U_{t}\right\}_{t \in \mathbb{R}}$ be an intrinsically random unitary dynamics with random image $\left\{W_{t}\right\}_{t \geq 0}$ on $L^{2}$. Then

a) $\left\{U_{t}\right\}_{t \in \mathbb{R}}$ is unitarily equivalent to the residual group $\left\{R_{t}\right\}_{t \in \mathbb{R}}$ of the minimal isometric dilation of $\left\{W_{t}\right\}_{t \geq 0}$

b) $\left\{W_{t \mid\left(L^{2} \ominus \mathbb{C}\right)}\right\}_{t \geq 0}$ is a contraction semigroup of class $C_{01}$, i.e.,

$$
\begin{aligned}
& \mathrm{s}-\lim _{t \rightarrow \infty} W_{t \mid\left(L^{2} \ominus \mathbb{C}\right)}=0 \text { and } \\
& \mathrm{s}-\lim W_{t \mid\left(L^{2} \ominus \mathbb{C}\right)}^{*} \rho \neq 0 \text { for every nonzero } \rho \in L^{2} \ominus \mathbb{C} \\
& (\text { asterisk } * \text { denotes adjoint }) .
\end{aligned}
$$

Also functional models may be given in terms of the characteristic function $\Theta_{W}$, a purely contractive analytic operator-valued function on the standard unit disc $D:=\{\lambda \in \mathbb{C} ;|\lambda|<1\}$. In order to define $\Theta_{W}$, let us consider the defect operators $D_{W}:=\left(I_{\mathcal{H}}-W^{*} W\right)^{1 / 2}$ and $D_{W^{*}}:=\left(I_{\mathcal{H}}-W W^{*}\right)^{1 / 2}$, and defect spaces $\mathcal{D}_{W}:=\overline{D_{W} L^{2}} \mathcal{D}_{W^{*}}:=\overline{D_{W^{*}} L^{2}}$ (overbar denotes adherence). The characteristic function of $W$ is defined by

$$
\Theta_{W}(\lambda):=\left[-W+\lambda D_{W^{*}}\left(I-\lambda W^{*}\right)^{-1} D_{W}\right]_{\mid \mathcal{D}_{W}}, \quad \lambda \in D .
$$

The non-tangential limits s- $\lim _{\lambda \rightarrow \omega} \Theta_{W}(\lambda)=: \Theta_{W}(\omega)$ exist for almost all $\omega$ in the boundary $\partial D$ and induce a decomposable operator $\left[\Theta_{W} v\right](\omega):=\Theta_{W}(\omega) v(\omega)$ 
from $L^{2}\left(\mathcal{D}_{W}\right)$ into $L^{2}\left(\mathcal{D}_{W^{*}}\right)$, where $L^{2}\left(\mathcal{D}_{W}\right)$ denotes the Hilbert space of square integrable $\mathcal{D}_{W}$-valued functions on $\partial D$ (with respect to the normalized Lebesgue measure and modulo sets of measure zero). Let $H^{2}\left(\mathcal{D}_{W}\right)$ denote the Hardy class of functions in $L^{2}\left(\mathcal{D}_{W}\right)$ whose kth Fourier coefficients vanish for all negative $\mathrm{k}$.

Corollary 2. Let $\left\{U_{t}\right\}_{t \in \mathbb{R}}$ be an intrinsically random unitary dynamics with random image $\left\{W_{t}\right\}_{t \geq 0}$ on $L^{2}$. Let $W$ be the cogenerator of $\left\{W_{t}\right\}_{t \geq 0}$. Then

a) $\left\{U_{t \mid\left(L^{2} \ominus \mathbb{C}\right)}\right\}_{t \in \mathbb{R}}$ is unitarily equivalent to the functional model

$$
\hat{\mathcal{R}}:=\overline{\Delta_{W} L^{2}\left(\mathcal{D}_{W}\right)}, \quad \hat{R}_{t}(v):=\exp \left(t \frac{\omega+1}{\omega-1}\right) v(\omega), \quad v \in \hat{\mathcal{R}}, t \in \mathbb{R}
$$

where $\Delta_{W}(\omega):=\left[I-\Theta_{W}(\omega)^{*} \Theta_{W}(\omega)\right]^{1 / 2}$

b) the characteristic function $\Theta_{W}$ of $W$ is outer and $*$-inner (see [10, Section V.2] for definitions of outer and $*$-inner functions), and

$$
\operatorname{ker} \Theta_{W} \cap H^{2}\left(\mathcal{D}_{W}\right)=\{0\} \text {. }
$$

Clear advantages derive from the existence of universal representatives, as for example determining spectral properties and relations. In the following result $\varepsilon\left(W_{t \mid\left(L^{2} \ominus \mathbb{C}\right)}\right)$ denotes the set of points $\omega \in \partial D$ at which $\Theta_{W_{t \mid\left(L^{2} \ominus \mathbb{C}\right)}}$ exists and is not isometric. Its essential support, denoted by "ess supp $\varepsilon\left(W_{t \mid\left(L^{2} \ominus \mathbb{C}\right)}\right)$ ", is defined as the complement with respect to $\partial D$ of the maximal open subset of $\partial D$ whose intersection with $\varepsilon\left(W_{t \mid\left(L^{2} \ominus \mathbb{C}\right)}\right)$ is of zero Lebesgue measure.

Corollary 3. Let $\left\{U_{t}\right\}_{t \in \mathbb{R}}$ be an intrinsically random unitary dynamics with random image $\left\{W_{t}\right\}_{t \geq 0}$ on $L^{2}$. Then

a) the spectrum $\sigma\left(W_{t \mid\left(L^{2} \ominus \mathbb{C}\right)}\right)$ of $W_{t \mid\left(L^{2} \ominus \mathbb{C}\right)}$ coincides with the set of points $\lambda \in D$ for which the operator $\Theta_{W_{t \mid\left(L^{2} \ominus \mathbb{C}\right)}}$ is not boundedly invertible, together with those $\omega \in \partial D$ not lying on any of the open arcs of $\partial D$ on which $\Theta_{W_{t \mid\left(L^{2} \ominus \mathbb{C}\right)}}$ is unitary. The point spectrum $\sigma_{p}\left(W_{t \mid\left(L^{2} \ominus \mathbb{C}\right)}\right)$ is the set of points $\lambda \in D$ for which $\Theta_{W_{t \mid\left(L^{2} \ominus \mathbb{C}\right)}}$ is not invertible at all.

b) $\sigma_{p}\left(U_{t}\right)=\sigma_{p}\left(W_{t}\right) \cap C=\{1\}$ and the eigenspace is $\mathbb{C}$, for every $t \geq 0$

c) $\sigma\left(U_{t \mid\left(L^{2} \ominus \mathbb{C}\right)}\right)=\sigma_{a c}\left(U_{t \mid\left(L^{2} \ominus \mathbb{C}\right)}\right)=$ ess supp $\varepsilon\left(W_{t \mid\left(L^{2} \ominus \mathbb{C}\right)}\right)$, for every $t \geq 0$. 


\section{Time Operator, Scattering and Innovation}

Following a suggestion by Misra [7], intrinsically random unitary evolutions $\left\{U_{t}\right\}$ on $L^{2}$ have been qualified by the existence of an internal time operator $T$, a selfadjoint operator satisfying the following relation: for every $\rho \in \operatorname{Dom}(T)$ (the domain of $T$ ) and $t \in \mathbb{R}$, one has $U_{t} \rho \in \operatorname{Dom}(T)$ and

$$
U_{-t} T U_{t} \rho=(T+t I) \rho .
$$

The time operator $T$ allows the attribution of an average age $(\rho, T \rho)$ to the states $\rho \in L^{2}$ which keeps step with the external clock time $t$ for the evolved state $U_{t} \rho$ : $\left(U_{t} \rho, T U_{t} \rho\right)=(\rho, T \rho)+t$, where $(\cdot, \cdot)$ denotes the inner product in $L^{2}$.

Actually, not only the expectation value but the entire probability distribution of ages is shifted by the external time, i.e., the time operator $T$ satisfy, for any $t \in \mathbb{R}$,

$$
U_{-t} E_{T}(\cdot) U_{t}=E_{T}(\cdot-t)
$$

where $E_{T}(\cdot)$ denotes the (projection-valued) spectral measure of $T$. Relation (3) is equivalent to (4) as well as to the usual Weyl commutation relation: if $A$ is the selfadjoint generator of the time evolution $\left\{U_{t}=\mathrm{e}^{\mathrm{i} t A}\right\}_{t \in \mathbb{R}}$, for all $s, t \in \mathbb{R}$,

$$
\mathrm{e}^{\mathrm{i} t A} \mathrm{e}^{\mathrm{i} s T}=\mathrm{e}^{\mathrm{i} t s} \mathrm{e}^{\mathrm{i} s T} \mathrm{e}^{\mathrm{i} t A} .
$$

Moreover, relation (3), (4) or (5) implies the Heisenberg or canonical commutation relation: for every $\rho \in \operatorname{Dom}(T A) \cap \operatorname{Dom}(A T)$

$$
[T, A] \rho:=T A \rho-A T \rho=\mathrm{i} \rho
$$

but the converse is not true in general.

It is also well known that every pair $(T, A)$ of selfadjoint operators satisfying (5) is unitarily equivalent to the momentum-position Schrödinger couple $(P, Q)$ on $L^{2}(\mathbb{R})$ or a direct sum of such couples. This is equivalent to the fact that $T$ and $A$ have absolutely continuous and uniform spectra spanning the entire real line.

The functional model given in Corollary 2 together with Halmos-Helson theory of invariant subspaces $[4,5]$ shed light on the connection between intrinsic randomness and internal time operators. For it, recall that a closed subspace $\mathcal{M}$ of $L^{2}\left(\mathcal{D}_{W}\right)$ is called invariant if $\omega \cdot v(\omega) \in \mathcal{M}$ for every $v \in \mathcal{M}, \mathcal{M}$ is called doubly invariant if $\omega \cdot v(\omega)$ and $\omega^{-1} \cdot v(\omega)$ belong to $\mathcal{M}$ for each $v \in \mathcal{M}$, and $\mathcal{M}$ is called simply invariant if it is invariant but not doubly invariant. On the other hand, let $\mathcal{L}\left(\mathcal{D}_{W}\right)$ denote the space of bounded linear operators on $\mathcal{D}_{W}$. A weakly measurable $\mathcal{L}\left(\mathcal{D}_{W}\right)$-valued function $U$ on $\partial D$ is called a rigid operator function if $U(\omega)$ is for almost all $\omega \in \partial D$ a partial isometry on $\mathcal{D}_{W}$ with the same initial space. 
Theorem 4. Let $\left\{U_{t}\right\}_{t \in \mathbb{R}}$ be a unitary dynamics on $L^{2}$. The following assertions are equivalent

a) there exists an internal time operator for $\left\{U_{t \mid\left(L^{2} \ominus \mathbb{C}\right)}\right\}_{t \in \mathbb{R}}$

b) $\left\{U_{t}\right\}_{t \in \mathbb{R}}$ is intrinsically random and, moreover, for the functional model $\left\{\hat{R}_{t}\right\}$ of $\left\{U_{t \mid\left(L^{2} \ominus \mathbb{C}\right)}\right\}$ given in Corollary 2.(a) there exists a simply invariant subspace $\hat{\mathcal{M}} \subseteq \hat{\mathcal{R}}$ of $L^{2}\left(\mathcal{D}_{W}\right)$ such that

$$
\bigcap_{n \in \mathbb{Z}} \hat{R}^{n} \hat{\mathcal{M}}=\{0\}, \quad \overline{\bigcup_{n \in \mathbb{Z}} \hat{R}^{n} \hat{\mathcal{M}}}=\hat{\mathcal{R}}
$$

where $\hat{R}$ is the cogenerator of $\left\{\hat{R}_{t}\right\}$ on $\hat{\mathcal{R}}$

c) $\left\{U_{t}\right\}_{t \in \mathbb{R}}$ is intrinsically random and, moreover, there exists a rigid $\mathcal{L}\left(\mathcal{D}_{W}\right)$ valued function $\hat{U}$ on $\partial D$ such that

$$
\overline{\bigcup_{n \in \mathbb{Z}} \hat{R}^{n} \hat{U} H^{2}\left(\mathcal{D}_{W}\right)}=\hat{\mathcal{R}}
$$

See [3] and references therein for details.

On the basis of Theorem 4, unitary dynamics $\left\{U_{t}\right\}$ without time operator but satisfying the intertwining relation (2) with dissipative evolutions $\left\{W_{t}\right\}$ have been constructed in [1].

It is also clear from Theorem 4.(b) that unitary evolutions $\left\{U_{t}\right\}_{t \in \mathbb{R}}$ with time operators are characterized by the existence of incoming and outgoing subspaces, i.e., closed subspaces $\mathcal{M}_{-}$and $\mathcal{M}_{+}$of $L^{2} \ominus \mathbb{C}$ with the following properties

$$
\begin{aligned}
& U_{t} \mathcal{M}_{ \pm} \subseteq \mathcal{M}_{ \pm}, \quad \text { for all } t \in \mathbb{R}^{ \pm} \\
& \bigcap_{t \in \mathbb{R}} U_{t} \mathcal{M}_{ \pm}=\{0\}, \quad \overline{\bigcup_{t \in \mathbb{R}} U_{t} \mathcal{M}_{ \pm}}=L^{2} \ominus \mathbb{C} .
\end{aligned}
$$

To see this, notice that the subspace $\mathcal{M}_{-} \subseteq L^{2} \ominus \mathbb{C}$ corresponding to the simply invariant subspace $\hat{\mathcal{M}} \subseteq \hat{\mathcal{R}}$ of the functional model is an incoming subspace for $\left\{U_{t \mid\left(L^{2} \ominus \mathbb{C}\right)}\right\}_{t \in \mathbb{R}}$. Since the existence of outgoing and incoming subspaces characterizes the unitary evolutions the Lax-Phillips scattering theory deals with [6], one has the following

Corollary 5. For every Lax-Phillips scattering process there exists an internal time operator and conversely. 
Distinguished translation and spectral representations are associated to different outgoing and incoming subspaces, so that representations derived from mutually orthogonal subspaces are related by means of a scattering operator $S$. The operator $S$ is usually interpreted as connecting the behavior in the remote past with that in the distant future.

Another type of dynamics with internal time operators are the purely nondeterministic innovative unitary evolutions [11] described by unitary groups $\left\{U_{t}\right\}_{t \in \mathbb{R}}$ for which there exists an increasing family of closed subspaces $\left\{\mathcal{M}_{t}\right\}_{t \in \mathbb{R}}$ such that

$$
U_{t_{1}} \mathcal{M}_{t_{2}}=U_{t_{2}} \mathcal{M}_{t_{1}}, \quad t_{1}, t_{2} \in \mathbb{R}, \quad \bigcap_{t \in \mathbb{R}} \mathcal{M}_{t}=\{0\} .
$$

Since the subspace $\mathcal{M}_{0}$ is an incoming subspace for such evolutions, one has the following:

Corollary 6. The purely nondeterministic innovative unitary evolutions are qualified by the existence of internal time operators and conversely.

Particular cases of such innovative processes are the so-called Kolmogorov flows, where the spectral projections of the internal time operator are coarse-graining projections of conditional expectation over the cells of the $K$-partition.

\section{Acknowledgments}

The authors wish to thank the XXV-th WGMP organizers for the kind hospitality. F. Gómez-Cubillo was supported by JCyL-project VA013C05 (Castilla y León) and MEC-project FIS2005-03989 (Spain).

\section{References}

[1] Gómez F., Non-unitary Similarity Transformation of Conservative to Dissipative Evolutions: Intertwining without Time Operator, J. Math. Phys. 48 (2007) 045201 (19 pages).

[2] Gómez F., Intrinsic Randomness of Unstable Dynamics and Sz.-Nagy-Foiaş Dilation Theory, preprint math-ph/0607031.

[3] Gómez F., Selfadjoint Time Operators and Invariant Subspaces, preprint math-ph/0607041. 
[4] Halmos P., Shifts on Hilbert Spaces, J. reine angew. math. 208 (1961) 102112.

[5] Helson H., Lectures on Invariant Subspaces, Academic Press, New York, 1964.

[6] Lax P. and Phillips R., Scattering Theory, Academic Press, New York, 1967.

[7] Misra B., Non-equilibrium Entropy, Lyapounov Variables and Ergodic Properties of Classical Systems, Proc. Natl. Acad. Sci. U.S.A. 75 (1978) 16271631.

[8] Misra B., Prigogine I. and Courbage M., From Deterministic Dynamics to Probabilistic Descriptions, Proc. Natl. Acad. Sci. U.S.A. 76 (1979) 36073611; Lyapounov Variable: Entropy and Measurement in Quantum Mechanics, Proc. Natl. Acad. Sci. U.S.A. 76 (1979) 4768-4772; From Deterministic Dynamics to Probabilistic Descriptions, Physica A98 (1979) 1-26.

[9] Pine D., Gollub J., Brady J. and Leshansky A., Chaos and Threshold for Irreversibility in Sheared Suspensions, Nature 438 (2005) 997-1000.

[10] Sz.-Nagy B. and Foiaş C., Harmonic Analysis of Operators on Hilbert Spaces, North-Holland, Amsterdam, 1970.

[11] Wold H., A Study in the Analysis of Stationary Time Series, Almqvist and Wiksell, Stockholm, 1938 (2nd Ed., 1954).

Fernando Gómez-Cubillo

Dept. de Análisis Matemático

Universidad de Valladolid

Facultad de Ciencias

Prado de la Magdalena, s.n.

47005 Valladolid

SPAIN

E-mail address: fgcubilleam.uva.es

Zdzislaw Suchanecki

Université du Luxembourg

Campus Kirchberg

6, Rue Coudenhove-Kalergi

L-1359 LUXEMBOURG

and

Institute of Mathematics and Informatics

University of Opole

POLAND

E-mail address: zdzislaw.suchanecki@uni.lu 